\title{
Oscillation Theorems for Second-Order Nonlinear Dynamic Equation on Time Scales
}

\author{
M.Tamer Şenel* \\ Department of Mathematics, Faculty of Sciences, Erciyes University, 38039, Kayseri, Turkey
}

Received: 27 Feb. 2013, Revised: 25 Jun. 2013, Accepted: 26 Jun. 2013

Published online: 1 Nov. 2013

Abstract: This paper concerns the oscillation of solutions to the second order non-linear dynamic equation

$$
\left(r(t) x^{\Delta}(t)\right)^{\Delta}+p(t) f\left(x^{\sigma}(t)\right) g\left(x^{\Delta}(t)\right)=0
$$

on a time scale $\mathbb{T}$ which is unbounded above. By using a generalized Riccati transformation and integral averaging technique, we establish some new sufficient conditions which ensure that every solution of this equation oscillates.

Keywords: Oscillation, Dynamic equations, Time scales, Second-order.

\section{Introduction}

In this paper, we consider the second order nonlinear dynamic equation

$$
\left(r(t) x^{\Delta}(t)\right)^{\Delta}+p(t) f\left(x^{\sigma}(t)\right) g\left(x^{\Delta}(t)\right)=0,
$$

where $p, r$ real-valued, non-negative, right-dense continuous function on a time scale $\mathbb{T}$. It is the purpose of this paper to give oscillation criteria for equation (1).

Throughout this paper, we will assume the following hypotheses:

(A1) $p, r \in C_{r d}\left(\left[t_{0}, \infty\right), \mathbb{R}^{+}\right)$such that $\int_{t_{0}}^{\infty} \frac{1}{r(s)} \Delta s=\infty$,

(A2) $g: \mathbb{T} \rightarrow \mathbb{R}^{+}$rd-continuous and $g(u) \geq c>0$,

(A3) $f: \mathbb{T} \rightarrow \mathbb{R}$ is continuously differentiable and satisfies $u f(u)>0, \frac{f(u)}{u} \geq k_{1}>0, u \neq 0$.

Much recent attention has been given to dynamic equations on time scales, or measure chains, and we refer the reader to the landmark paper of Hilger [12] for a comprehensive treatment of the subject. Since then, several authors have expounded on various aspects of this new theory; see the survey paper by Agarwal et al. [1] and the references cited therein. A book on the subject of time scales by Bohner and Peterson [3] summarizes and organizes much of the time scale calculus. In recent years there has been much research activity concerning the oscillation and nonoscillation of solutions of some different equations on time scales. We refer the reader to the papers $[2,4-6,8,11]$. In [6], the authors consider the second order dynamic equation

$$
\left(p(t) x^{\Delta}(t)\right)^{\Delta}+q(t) x^{\sigma}(t)=0 \text { for } t \in[a, b]
$$

and give necessary and sufficient conditions for oscillation of all solutions on unbounded time scales. Unfortunately, the oscillation criteria are restricted in usage since additional assumptions have to be imposed on the unknown solutions. In [10], the authors consider the same equation and suppose that there exists some $t_{0} \in T$ such that $\mathrm{p}$ is bounded above on $\left[t_{0}, \infty\right)$, $\inf \left\{\mu(t): t \in\left[t_{0}, \infty\right)\right\}>0$ and use the Riccati equation to prove that if

$$
\int_{t_{0}}^{\infty} q(t) \Delta t=\infty
$$

then every solution is oscillatory on $\left[t_{0}, \infty\right)$.

In [5], the authors consider

$$
\left(p(t) x^{\Delta}(t)\right)^{\Delta}+q(t)\left(\text { fox }^{\sigma}\right)=0 \text { for } t \in[a, b],
$$

where $p$ and $q$ are positive, real-valued rd-continuous functions.

\footnotetext{
*Corresponding author e-mail: senel@erciyes.edu.tr, tsenel@gmail.com
} 


\section{Preliminary results}

Lemma 2.1. Let $x(t)$ be a nonoscillatory solution of (1) and (A1)-(A3) hold. Then there exist $T_{0}$ such that

$$
x(t)>0, x^{\Delta}(t)>0, \quad\left(r(t) x^{\Delta}(t)\right)^{\Delta}<0 \text { for } t \geq T_{0} .
$$

Proof. Suppose that $x(t)$ is nonoscillatory solution of (1) and without loss of generality, assume $x(t)>0$ for $t \geq T_{0}$. Assume that $x^{\Delta}(t)<0$ for all large $t$. Then without loss of generality $x^{\Delta}(t)<0$ for all $t \geq T_{1} \geq T_{0}$. If $x(t)>0$, then $f\left(x^{\sigma}(t)\right)>0$. From (1)

$$
\left(r(t) x^{\Delta}(t)\right)^{\Delta}=-p(t) f\left(x^{\sigma}(t)\right) g\left(x^{\Delta}(t)\right)<0,
$$

$\left(r(t) x^{\Delta}(t)\right)^{\Delta}<0$.

Define $y(t)=r(t) x^{\Delta}(t)$. So $y(t)$ is decreasing. Assume that there exist $T_{1} \geq T_{0}$ with $y\left(T_{1}\right)=\kappa<0$. Then

$$
r(t) x^{\Delta}(t)=y(t) \leq y\left(T_{1}\right)=\kappa \text { for all } t \geq T_{1}
$$

and therefore

$$
x^{\Delta}(t) \leq \frac{\kappa}{r(t)} \text { for all } t \geq T_{1}
$$

Then an integration for $t>T_{2} \geq T_{1}$ gives

$x(t) \leq x\left(T_{2}\right)+\kappa \int_{T_{2}}^{t} \frac{1}{r(s)} \Delta s \rightarrow-\infty$ as $t \rightarrow \infty$

which is a contradiction. Hence $x^{\Delta}(t)$ is not negative for all large $t$ and thus $x^{\Delta}(t)>0$ for all $t \geq T_{0}$.

\section{Main results}

Theorem 3.1 Assume that (A1)-(A3) hold. Furthermore, assume that there exist a positive real rd-functions differentiable functions $z(t)$ such that

$$
\limsup _{t \rightarrow \infty} \int_{T}^{t}\left[k_{1} c z(s) p(s)-\frac{1}{4} \frac{\left(z^{\Delta}(t)\right)^{2}}{z(s)}\right] \Delta s=\infty,
$$

then every solution of (1) is oscillatory.

Proof. Suppose to the contrary that $x(t)$ is a nonoscillatory solution of (1). Without loss of generality, we may assume that $x(t)>0$ for $t \geq T_{1}>T_{0}$. We shall consider only this case, the proof of the case when $\mathrm{x}(\mathrm{t})$ is eventually negative is similar. From Lemma 2.1, $x^{\Delta}(t)>0$ for $t \geq T_{1}>T_{0}$. Define the function $w(t)$ by the Riccati substitution

$$
w(t):=z(t) \frac{r(t) x^{\Delta}(t)}{x(t)}, t \geq T_{1} .
$$

Then $w(t)>0$ satisfies

$$
w^{\Delta}(t)=\left[\frac{z(t)}{x(t)}\right]^{\Delta}\left(r(t) x^{\Delta}(t)\right)^{\sigma}+\frac{z(t)}{x(t)}\left(r(t) x^{\Delta}(t)\right)^{\Delta}
$$

$$
\begin{aligned}
w^{\Delta}(t)= & \frac{z^{\Delta}(t) x(t)-z(t) x^{\Delta}(t)}{x(t) x^{\sigma}(t)}\left(r(t) x^{\Delta}(t)\right)^{\sigma} \\
+ & \frac{z(t)}{x(t)}\left(-p(t) f\left(x^{\sigma}(t)\right) g\left(x^{\Delta}(t)\right)\right) .
\end{aligned}
$$

From Lemma 2.1, $x(t)>0, x^{\Delta}(t)>0,\left(r(t) x^{\Delta}(t)\right)^{\Delta}<0$ so

$$
x^{\sigma}(t)>x(t),\left(r(t) x^{\Delta}(t)\right)^{\sigma}<r(t) x^{\Delta}(t) .
$$

We get

$$
\begin{aligned}
w^{\Delta}(t) \leq z^{\Delta}(t) \frac{w^{\sigma}(t)}{z^{\sigma}(t)} & -z(t) \frac{x^{\Delta}(t)\left(r(t) x^{\Delta}(t)\right)^{\sigma}}{\left(x^{\sigma}(t)\right)^{2}} \\
& -\frac{z(t)}{x^{\sigma}(t)} p(t) f\left(x^{\sigma}(t)\right) c
\end{aligned}
$$

$$
\begin{aligned}
w^{\Delta}(t) \leq & -k_{1} c z(t) p(t)+z^{\Delta}(t) \frac{w^{\sigma}(t)}{z^{\sigma}(t)} \\
& -z(t) \frac{x^{\Delta}(t)\left(w^{\sigma}(t)\right)^{2}}{\left.\left(z^{\sigma}(t)\right)^{2}\left(r(t)\left(x^{\Delta}(t)\right)\right)^{\sigma}\right)}
\end{aligned}
$$

$$
\leq-k_{1} c z(t) p(t)+z^{\Delta}(t) \frac{w^{\sigma}(t)}{z^{\sigma}(t)}-z(t) \frac{\left(w^{\sigma}(t)\right)^{2}}{\left(z^{\sigma}(t)\right)^{2} r^{\sigma}(t)}
$$

$w^{\Delta}(t) \leq-k_{1} c z(t) p(t)+\frac{1}{4} \frac{r^{\sigma}(t)\left(z^{\Delta}(t)\right)^{2}}{z(t)}-\left[\sqrt{\frac{z(t)}{r^{\sigma}(t)}} \frac{w^{\sigma}(t)}{z^{\sigma}(t)}-\frac{1}{2} \sqrt{\frac{r^{\sigma}(t)}{z(t)}} z^{\Delta}(t)\right]^{2}$,

then

$w^{\Delta}(t) \leq-k_{1} c z(t) p(t)+\frac{1}{4} \frac{r^{\sigma}(t)\left(z^{\Delta}(t)\right)^{2}}{z(t)}$.

Integrating (8) from $T \geq T_{1}$ to $t$

$w(t)-w(T) \leq-\int_{T}^{t}\left[k_{1} c z(t) p(t)-\frac{1}{4} \frac{r^{\sigma}(t)\left(z^{\Delta}(t)\right)^{2}}{z(t)}\right] \Delta s$,

$\int_{T}^{t}\left[k_{1} c z(t) p(t)-\frac{1}{4} \frac{r^{\sigma}(t)\left(z^{\Delta}(t)\right)^{2}}{z(t)}\right] \Delta s \leq w(T)-w(t)<w(T)$.

Taking the limsup of both sides of above inequality as $t \rightarrow \infty$, we obtain a contradiction to condition (3). The proof is complete.

Corollary 3.2. Assume that (A1)-(A3) hold. If

$$
\limsup _{t \rightarrow \infty} \int_{T}^{t} k_{1} c p(s) \Delta s=\infty,
$$

then every solution (1) is oscillatory.

Example 3.3. Consider the dynamic equation

$\left(t x^{\Delta}(t)\right)^{\Delta}+\frac{1}{\mu(t)} x^{\sigma}(t)\left(1+\left(x^{\sigma}(t)\right)^{2}\right)\left(1+\left(x^{\Delta}(t)\right)^{2}\right)=0, t \in \mathbb{T}$.

where

$r(t)=t, p(t)=\frac{1}{\mu(t)}, f\left(x^{\sigma}(t)\right)=x^{\sigma}(t)\left(1+\left(x^{\sigma}(t)\right)^{2}\right)$, $g\left(x^{\Delta}(t)\right)=1+\left(x^{\Delta}(t)\right)^{2}$.

If we take :

i) $\mathbb{T}=\mathbb{Z} \Rightarrow \sigma(t)=t \Rightarrow \mu(t)=1 \Rightarrow p(t)=1$,

ii) $\mathbb{T}=\left\{2^{N}: n \in \mathbb{Z}\right\} \cup\{0\} \Rightarrow \sigma(t)=2 t \Rightarrow \mu(t)=t \Rightarrow$ 
$p(t)=\frac{1}{t}$

all conditions of Corollary 3.2 are satisfied. Hence it is oscillatory.

Corollary 3.4. Assume that (A1)-(A3) hold. If there is $\lambda \geq 1$ such that

$$
\limsup _{t \rightarrow \infty} \int_{T}^{t}\left[k_{1} c s^{\lambda} p(s)-\frac{1}{4} \frac{r^{\sigma}(t)\left(\left(s^{\lambda}\right)^{\Delta}\right)^{2}}{s^{\lambda}}\right] \Delta s=\infty,
$$

then every solution (1) is oscillatory.

Now, let us introduce the class of functions $\mathbb{R}$ which will be extensively used in the sequel. Let $\mathbb{D}_{0} \equiv\left\{(t, s) \in \mathbb{T}^{2}: t>s \geq t_{0}\right\} \quad$ and $\mathbb{D} \equiv\left\{(t, s) \in \mathbb{T}^{2}: t \geq s \geq t_{0}\right\}$. The function $H \in C_{r d}(\mathbb{D}, \mathbb{R})$ is said belong to the class $\mathfrak{R}$ if

(i) $H(t, t)=0, t \geq t_{0}, H(t, s)>0$, on $\mathbb{D}_{0}$,

(ii) $\mathrm{H}$ has a continuous $\Delta$-partial derivative $H_{s}^{\Delta}(t, s)$ on $\mathbb{D}_{0}$ with respect to the second variable. ( $\mathrm{H}$ is rd-continuous function if $\mathrm{H}$ is rd-continuous function in $\mathrm{t}$ and $\mathrm{s}$.)

Theorem 3.6. Assume that (A1)-(A3) hold. Let $\mathrm{z}(\mathrm{t})$ be a positive differentiable function and let $H: \mathbb{D} \rightarrow \mathbb{R}$ be an rd-continuous function such that $\mathrm{H}$ belongs to the class $\mathfrak{R}$ and satisfies

$\limsup _{t \rightarrow \infty} \frac{1}{H(t, T)} \int_{T}^{t}\left[k_{1} c H(t, s) z(s) p(s)-\frac{1}{4} C(t, s)\right] \Delta s=\infty$,

where

$C(t, s)=r^{\sigma}(s)\left(z^{\sigma}(s) B(t, s)\right)^{2}$,

$B(t, s)=H_{s}^{\Delta}(t, s)+H(t, s) \frac{z^{\Delta}(s)}{z^{\sigma}(s)}$.

Then every solution of (1) is oscillatory.

Proof. Suppose to the contrary that $x(t)$ is a nonoscillatory solution of (1). Without loss of generality, we may assume that $x(t)>0$ for $t \geq T_{1}>T_{0}$. We shall consider only this case, the proof of the case when $x(t)$ is eventually negative is similar. From Lemma 2.1 and (7), $x^{\Delta}(t)>0$ for $t \geq T_{1}>T_{0}$, it follows that

$$
\begin{aligned}
w^{\Delta}(t) \leq-k_{1} c z(t) p(t) & +z^{\Delta}(t) \frac{w^{\sigma}(t)}{z^{\sigma}(t)} \\
& -z(t) \frac{\left(w^{\sigma}(t)\right)^{2}}{\left(z^{\sigma}(t)\right)^{2} r^{\sigma}(t)} .
\end{aligned}
$$

We multiply both sides by $H(t, s)$ to get

$$
\begin{aligned}
H(t, s) w^{\Delta}(t) \leq & -k_{1} c H(t, s) z(t) p(t)+H(t, s) z^{\Delta}(t) \frac{w^{\sigma}(t)}{z^{\sigma}(t)} \\
& -H(t, s) z(t) \frac{\left(w^{\sigma}(t)\right)^{2}}{\left(z^{\sigma}(t)\right)^{2} r^{\sigma}(t)} .
\end{aligned}
$$

Using the integration by parts formula, we have

$$
\begin{array}{rlr}
\int_{T}^{t} k_{1} c H(t, s) z(s) p(s) \Delta s \leq-H(t, t) w(t) & + & H(t, T) w(T) \\
& + & \int_{T}^{t} H_{s}^{\Delta}(t, s) w^{\sigma}(s) \Delta s \\
& + & \int_{T}^{t} H(t, s) z^{\Delta}(s) \frac{w^{\sigma}(s)}{z^{\sigma}(s)} \Delta s \\
& -\int_{T}^{t} H(t, s) z(s) \frac{\left(\left(w^{\sigma}(s)\right)^{2}\right.}{\left(z^{\sigma}(s)\right)^{2} r^{\sigma}(s)} \Delta s
\end{array}
$$

from $H(t, t)=0$, we obtain

$$
\begin{array}{rlr}
\int_{T}^{t} k_{1} c H(t, s) z(s) p(s) \Delta s \leq & H(t, T) w(T) \\
& +\int_{T}^{t}\left[H_{s}^{\Delta}(t, s)+H(t, s) \frac{z^{\Delta}(s)}{z^{\sigma}(s)}\right] w^{\sigma}(s) \Delta s \\
& - & \int_{T}^{t} H(t, s) z(s) \frac{\left(\left(w^{\sigma}(s)\right)^{2}\right.}{\left(z^{\sigma}(s)\right)^{2} r^{\sigma}(s)} \Delta s, \\
\int_{T}^{t} k_{1} c H(t, s) z(s) p(s) \Delta s \leq H(t, T) w(T)+ & \int_{T}^{t} B(t, s) w^{\sigma}(s) \Delta s \\
& -\int_{T}^{t} H(t, s) z(s) \frac{\left(\left(w^{\sigma}(s)\right)^{2}\right.}{\left(z^{\sigma}(s)\right)^{2} r^{\sigma}(s)} \Delta s .
\end{array}
$$

Therefore, by completing the square as in Theorem 3.1, we obtain

$\int_{T}^{t} k_{1} c H(t, s) z(s) p(s) \Delta s \leq H(t, T) w(T)+\int_{T}^{t} \frac{1}{4} r^{\sigma}(s)\left(z^{\sigma}(s)\right)^{2} B^{2}(t, s) \Delta s$

$-\int_{T}^{t}\left[\sqrt{\frac{H(t, s) z(s)}{r^{\sigma}(s)}} \frac{w^{\sigma}(s)}{z^{\sigma}(s)}-\frac{1}{2} \sqrt{\frac{r^{\sigma}(s)}{H(t, s) z(s)}} B(t, s) z^{\sigma}(s)\right]^{2} \Delta s$.

Hence, we obtain

$$
\begin{aligned}
\int_{T}^{t} k_{1} c H(t, s) z(s) p(s) \Delta s & \leq & H(t, T) w(T)+\int_{T}^{t} \frac{1}{4} r^{\sigma}(s)\left(z^{\sigma}(s) B(t, s)\right)^{2} \Delta s \\
& = & H(t, T) w(T)+\int_{T}^{t} \frac{1}{4} C(t, s) \Delta s
\end{aligned}
$$

where

$C(t, s)=r^{\sigma}(s)\left(z^{\sigma}(s) B(t, s)\right)^{2}$.

Then for all $t \geq T$, we have

$\int_{T}^{t}\left[k_{1} c H(t, s) z(s) p(s)-\frac{1}{4} C(t, s)\right] \Delta s \leq H(t, T) w(T)$

and this implies that

$\limsup _{t \rightarrow \infty} \frac{1}{H(t, T)} \int_{T}^{t}\left[k_{1} c H(t, s) z(s) p(s)-\frac{1}{4} C(t, s)\right] \Delta s \leq w(T)$

which contradicts (11). The proof is complete.

As consequences of Theorem 3.6 we get the following.

Corollary 3.7. Suppose that the assumptions of Theorem 3.6 hold. If

$\limsup _{t \rightarrow \infty} \frac{1}{H(t, T)} \int_{T}^{t}\left[k_{1} c H(t, s) p(s)-\frac{1}{4} r^{\sigma}(s)\left(H_{s}^{\Delta}(t, s)\right)^{2}\right] \Delta s=\infty$,

then every solution of (1) is oscillatory. 
Corollary 3.8. Let the assumption (11) in Theorem 3.6 be replaced by

$\limsup _{t \rightarrow \infty} \frac{1}{H(t, T)} \int_{T}^{t} k_{1} c H(t, s) z(s) p(s)=\infty$,

$\limsup _{t \rightarrow \infty} \frac{1}{H(t, T)} \int_{T}^{t}\left[\frac{r^{\sigma}(s)\left(z^{\sigma}(s)\right)^{2}}{4}\left(\frac{H(t, s) z^{\Delta}(s)}{z^{\sigma}(s)}+H_{s}^{\Delta}(t, s)\right)^{2}\right] \Delta s<\infty$.

Then every solution of (1) is oscillatory.

Lemma 3.9. Let $H(t, s)=(t-s)^{n},(t, s) \in \mathbb{D}$ with $n>1$, we see that $H$ belongs to the class $\mathfrak{R}$. Hence

$\left((t-s)^{n}\right)^{\Delta} \leq-n(t-\sigma(s))^{n-1}$.

Proof. We consider the following two cases:

Case 1. If $\mu(t)=0$ then

$$
\left((t-s)^{m}\right)^{\Delta}=-m(t-s)^{m-1} .
$$

Case 2. If $\mu(t) \neq 0$, then we have

$$
\begin{aligned}
\left((t-s)^{m}\right)^{\Delta} & =\frac{1}{\mu(s)}\left[(t-\sigma(s))^{m}-(t-s)^{m}\right] \\
& =-\frac{1}{\sigma(s)-s}\left[(t-s)^{m}-(t-\sigma(s))^{m}\right] .
\end{aligned}
$$

Using the Hardy, Littlewood and Polya inequality $x^{m}-y^{m} \geq \gamma y^{m-1}(x-y)$ for all $x \geq y>0$ and $m \geq 1$, we have

$\left[(t-s)^{m}-(t-\sigma(s))^{m}\right] \geq m(t-\sigma(s))^{m-1}(\sigma(s)-s)$.

Then, from (14) and (15), we have

$$
\left((t-s)^{m}\right)^{\Delta} \leq-m(t-\sigma(s))^{m-1} .
$$

Corollary 3.10. Assume that (A1)-(A3) hold. Let $z(t)=1$ $, H: \mathbb{D} \rightarrow \mathbb{R}$ be rd-continuous function such that $\mathrm{H}$ belongs to the class $\mathfrak{R}$. If

$\limsup t \rightarrow \infty \frac{1}{t^{n}} \int_{T}^{t}\left[k_{1} c(t-s)^{n} p(s)-\frac{r^{\sigma}(s)}{4}\left(n(t-\sigma(s))^{n-1}\right)^{2}\right] \Delta s=\infty$, for $n>1$, then equation (1) is oscillatory on $\left[t_{0}, \infty\right)$.

\section{Assume that $f$ is differentiable.}

In this section, we assume that $f^{\prime}(u) \geq k_{2}$ for $u \neq 0$ and some $k_{2}>0$.

Theorem 4.1. Assume that (A1)-(A3) hold. Furthermore, assume that there exists a positive real rd-continuous function $z(t)$ such that

$$
\limsup _{t \rightarrow \infty} \int_{T}^{t}\left[c z(s) p(s)-\frac{1}{4} \frac{r(s)\left(z^{\Delta}(t)\right)^{2}}{k_{2} z(s)}\right] \Delta s=\infty,
$$

then every solution of (1) is oscillatory.

Proof. Suppose to the contrary that $x(t)$ is a nonoscillatory solution of (1). Without loss of generality, we may assume that $x(t)>0$ for $t \geq T_{1}>T_{0}$. We shall consider only this case, the proof of the case when $x(t)$ is eventually negative is similar. From Lemma 2.1, $x^{\Delta}(t)>0$ for $t \geq T_{1}>T_{0}$. Define the function $w(t)$ by

$w(t):=z(t) \frac{r(t) x^{\Delta}(t)}{f(x(t))}, t \geq T_{1}$.

Then $w(t)$ satisfies

$w^{\Delta}(t)=\left(r(t)\left(x^{\Delta}(t)\right)\right)^{\sigma}\left[\frac{z(t)}{f(x(t))}\right]^{\Delta}+\frac{z(t)}{f(x(t))}\left(r(t) x^{\Delta}(t)\right)^{\Delta}$.

In view of (1), $f\left(x^{\sigma}\right) \geq f(x)$ and Lemma 2.1, we have

$$
\begin{aligned}
w^{\Delta}(t) \leq z^{\Delta}(t) \frac{\left(r(t) x^{\Delta}(t)\right)^{\sigma}}{f\left(x^{\sigma}(t)\right)} & -z(t) \frac{f^{\Delta}(x(t))\left(r(t) x^{\Delta}(t)\right)^{\sigma}}{f^{2}\left(x^{\sigma}(t)\right)} \\
& -c z(t) p(t) \frac{f\left(x^{\sigma}(t)\right)}{f\left(x^{\sigma}(t)\right)}
\end{aligned}
$$

$w^{\Delta}(t) \leq-c z(t) p(t)+z^{\Delta}(t) \frac{w^{\sigma}(t)}{z^{\sigma}(t)}-\frac{z(t)}{\left(z^{\sigma}(t)\right)^{2}} \frac{f^{\Delta}(x(t))\left(w^{\sigma}(t)\right)^{2}}{\left(r(t) x^{\Delta}(t)\right)^{\sigma}}$.

Using chain rule [4]

$f^{\Delta}(x(t))=f^{\prime}(x(\tau)) x^{\Delta}(t) \geq k_{2} x^{\Delta}(t), \tau \in[t, \sigma(t)]$.

We have

$w^{\Delta}(t) \leq-c z(t) p(t)+z^{\Delta}(t) \frac{w^{\sigma}(t)}{z^{\sigma}(t)}-\frac{z(t)}{\left(z^{\sigma}(t)\right)^{2}} \frac{k_{2} x^{\Delta}(t)\left(w^{\sigma}(t)\right)^{2}}{r(t) x^{\Delta}(t)}$

$\leq-c z(t) p(t)+z^{\Delta}(t) \frac{w^{\sigma}(t)}{z^{\sigma}(t)}-\frac{z(t)}{\left(z^{\sigma}(t)\right)^{2}} \frac{k_{2}\left(w^{\sigma}(t)\right)^{2}}{r(t)}$

$\leq-c z(t) p(t)+\frac{1}{4} \frac{r(t)\left(z^{\Delta}(t)\right)^{2}}{k_{2} z(t)}-\left[\sqrt{\frac{k_{2} z(t)}{r(t)}} \frac{w^{\sigma}(t)}{z^{\sigma}(t)}-\frac{1}{2} \sqrt{\frac{r(t)}{k_{2} z(t)}} z^{\Delta}(t)\right]^{2}$, then

$w^{\Delta}(t) \leq-c z(t) p(t)+\frac{1}{4} \frac{r(t)\left(z^{\Delta}(t)\right)^{2}}{k_{2} z(t)}$.

We proceed as in the proof of Theorem 3.1 and we obtain a contradiction.

Corollary 4.2. Assume that (A1)-(A3) hold. If

$$
\limsup _{t \rightarrow \infty} \int_{T}^{t}\left[c s^{\lambda} p(s)-\frac{r(t)\left(\left(s^{\lambda}\right)^{\Delta}\right)^{2}}{4 k_{2} r(s)}\right] \Delta s=\infty, \quad \lambda \geq 1,
$$

then every solution of (1) is oscillatory.

Different choices of $\mathrm{z}(\mathrm{t})$ lead to different corollaries of the above theorem.

Theorem 4.3. Assume that (A1)-(A3) hold. Let $\mathrm{z}(\mathrm{t})$ be a positive differentiable function and let $H: \mathbb{D} \rightarrow \mathbb{R}$ be an rd-continuous function such that $\mathrm{H}$ belongs to the class $\mathfrak{R}$ and

$\limsup _{t \rightarrow \infty} \frac{1}{H(t, T)} \int_{T}^{t}\left[c H(t, s) z(s) p(s)-\frac{r(s)}{4 k_{2} z(s) H(t, s)} E^{2}(t, s)\right] \Delta s=\infty$,

where

$E(t, s)=H(t, s) z^{\Delta}(t)+z^{\sigma}(t) H_{s}^{\Delta}(t, s)$. 
Then every solution of (1) is oscillatory.

Proof. The proof is similar to that of Theorem 3.6 and hence is omitted.

As an immediate consequence of Theorem 4.3 using $z(t)=1, H(t, s)=(t-s)^{m}$ and $m=n-1$, we get the following two results.

Corollary 4.4. Assume that (A1)-(A3) hold. The condition in Theorem 4.3 is replaced by

$$
\limsup _{t \rightarrow \infty} \frac{1}{H(t, T)} \int_{T}^{t} c H(t, s) z(s) p(s) \Delta s=\infty
$$

and

$\lim \sup _{t \rightarrow \infty} \frac{1}{H(t, T)} \int_{T}^{t} \frac{r(s)\left(H_{s}^{\Delta}(t, s) z^{\sigma}(s)+H(t, s) z^{\Delta}(s)\right)^{2}}{k_{2} H(t, s) z(s)} \Delta s<\infty$, then every solution of (1) is oscillatory on $\left[t_{0}, \infty\right)$.

Corollary 4.5. Assume that (A1)-(A3) hold. If for $n>2$

$$
\limsup _{t \rightarrow \infty} \frac{1}{t^{n-1}} \int_{T}^{t} c(t-s)^{n-1} p(s) \Delta s=\infty
$$

and

$\limsup \sup _{t \rightarrow \infty} \frac{1}{t^{n-1}} \int_{T}^{t} \frac{r(s)\left((n-1)(t-\sigma(s))^{n-2}\right)^{2}}{4 k_{2}(t-s)^{n-1}} \Delta s<\infty, t \geq s \geq T$, then every solution of (1) is oscillatory on $[T, \infty)$.

\section{Acknowledgement}

This work was supported by Research Fund of the Erciyes University. Project Number: FBA-11-3391.

The author is grateful to the anonymous referee for a careful checking of the details and for helpful comments that improved this paper.

\section{References}

[1] R. P. Agarwal, M. Bohner, D. O'Regan and A. Peterson, Dynamic equations on time scales: A survey, J. Comput. Appl. Math., 141, 1-26 (2002).

[2] E. Akın, L. Erbe, B. Kaymakçalan and A. Peterson, Oscillation results for a dynamic equation on a time scale, J. Differ. Equations Appl., 7, 793- 810 (2001).

[3] M. Bohner, A. Peterson, Dynamic Equations on Time Scales: An Introduction with Applications, Birkhäuser, Boston, (2001).

[4] M. Bohner, L. Erbe, A. Peterson, Oscillation for second order dynamic equations on time scale, J. Math. Anal. Appl., 301, 491-507 (2005).
[5] M. Bohner, S. H. Saker, Oscillation of second order nonlinear dynamic equations on time scales, Rocky Mountain J. Math., 34, 1239-1254 (2004).

[6] O. Došlý and S. Hilger, A necessary and sufficient condition for oscillation of the Sturm-Liouville dynamic equation on time scales, J. Comput. Appl. Math., 141, 147-158 (2002).

[7] Yuri V. Rogovchenko, Oscillation Criteria for Certain Nonlinear Differential Equations, J. Math. Anal. Appl., 229, 399-416 (1999).

[8] O. Došlý and R. Hilscher, Disconjugacy, transformations and quadratic functionals for symplectic dynamic systems on time scales, J. Differ. Equations Appl., 7, 265-295 (2001).

[9] L. Erbe and A. Peterson, Positive solutions for a nonlinear differential equation on a measure chain, in Boundary value problems and related topics, Math. Comput. Modelling, 32, 571-585 (2000).

[10] L. Erbe and A. Peterson, Riccati equations on a measure chain, in Proceedings of dynamic systems and applications (G. S. Ladde, N. G. Medhin and M. Sambandham, eds.), Dynamic Publishers, Atlanta, GA, 3, 193-199 (2001) .

[11] G. Sh. Guseinov and B. Kaymakçalan, On a disconjugacy criterion for second order dynamic equations on time scales, J. Comput. Appl. Math., 141, 187-196 (2002).

[12] S. Hilger, Analysis on measure chains A unified approach to continuous and discrete calculus, Results Math., 18, 18-56 (1990).

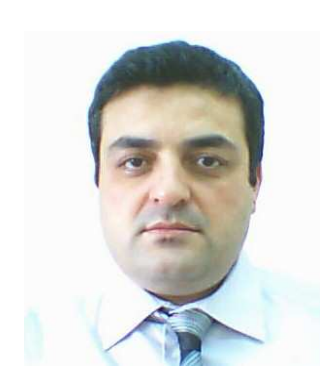

$\begin{array}{cr}\text { M. Tamer } & \text { Şenel } \\ \text { Associate } & \text { Professor }\end{array}$ Department of Mathematics of Faculty of Science at Erciyes University. His research interests are in the areas of applied mathematics and oscillation theory of differential and dynamic equations. He is referee and editor of mathematical journals. 